\title{
Gene expression database scales up, providing baseline data
}

The rise of genome-wide association studies put a spotlight on how small variations in DNA can affect a person's risk of disease. Exactly how these small changes influence the production of proteins is unclear; notably, up to $90 \%$ of the variants discovered through genome-wide association studies occur outside the protein coding regions of genes. So, as early as 2005 , Francis Collins, then director of the US National Human Genome Research Institute (NHGRI) in Bethesda, Maryland, championed the concept of the Genotype-Tissue Expression project (GTEx) to create a reference database of genetic expression in healthy human tissue. A 2008 workshop confirmed the feasibility Collins's idea, and in October 2010 the US National Institutes of Health (NIH) Common Fund finally launched the project, as Collins ascended to lead the NIH.

On 29 May, after a two-year pilot period, researchers behind GTEx released a progress update about how the database has evolved and how it promises to serve as a useful resource going forward (Nat. Genet. 45, 580-585, 2013). The project has already collected data on genetic expression from more than 1,800 postmortem tissue samples from a total of 190 healthy donors.

According to GTEx program director, the NHGRI's Jeff Streuwing, the database is the first to analyze levels of RNA in different tissues within an individual donor-24 different tissues per person, on average. In the two months following the official April launch of the GTEx database, about 55 researchers requested access.

One draw of the database is that scientists who study specific gene mutations or single nucleotide polymorphisms (SNPS) "can go put in that SNP number and see how that SNP acts in local tissues," Struewing says. Researchers must understand normal gene expression to grasp which signals go awry in illness, explains Kristin Ardlie, one of several principal investigators of the GTEx project and director of the Biological Samples Platform at the Broad Institute in Cambridge, Massachusetts. The goal is for genetic understanding to yield more effective disease therapeutics, Ardlie says, and the research GTEx allows is "the next step along that pathway to understand the function and regulation" of genetic expression.
Stephen Hewitt studies malignant tissue samples in his pathology lab, so the genetic expression data he sees primarily come from diseased cells. The GTEx database will bring him "a catalog of gene expression in a normal tissue," which is "incredibly valuable," says Hewitt, a cancer researcher at the US National Cancer Institute in Bethesda, Maryland, who helped develop the method GTEx uses to preserve its samples but has not been involved in data collection.

GTEx might also provide a baseline of RNA levels for researchers to understand how therapies influence tissue differently.

Researchers already make "use of RNA sequence data in the prediction of drug responses," Ardlie says. She points to a recent study that related the gene expression in cancer cell lines to their drug response; for example, that expression of the Schlafen family member 11 gene (SLFN11) predicted sensitivity to topoisomerase inhibitors (Nature 483, 603-607, 2012).

Last year, at the American Society of Human Genetics annual meeting in San Francisco, GTEx researchers reported that preliminary analysis of gene expression information in the database from blood, lung, thyroid, heart, muscle and skin tissue confirmed the presence of SNP epicenters regulating RNA levels known as 'expression quantitative trait loci' (eQTLs). Moreover, it showed the potential to pinpoint new eQTLs. Past research, for example, has pointed to an eQTL for orosomucoid like 3 (ORMDL3), a gene thought to influence asthma risk, though researchers haven't yet detected a difference in expression between diseased and healthy individuals (Nat. Rev. Genet. 12, 277-282, 2012).

Now that the pilot phase has ended, GTEx has begun to scale up-with the goal of offering gene expression data from 900 donors by 2015 . Down the road, the GTEx team hopes to run wholegenome methylation analysis on its samples, which would give more information on underlying biological mechanisms of gene expression, and proteomic analysis, which would provide insight on the proteins at work in each cell, Struewing says.

Susan Matthews
By comparison, the AIDS Clinical Trials Group, the major NIH clinical research organization for HIV/AIDS, is expecting a maximum $6 \%$ cut in funding, according to a source at the US National Institute of Allergy and Infectious Diseases. Though less consolidated than other infectious disease research in the US, malaria programs are also facing smaller cuts. The Parasitic Diseases and Malaria program at the CDC Center for Global Health-which includes malaria control as well as research-will be cut by $5.2 \%$,according to information provided by the US National Institute of Allergy and Infectious Diseases.

Clinical sites like those of the TBTC fostered the development of new tuberculosis treatments, which are necessary to combat a pandemic disease that increasingly shows drug resistance. As of 2012, there were more than 70 drugs in the clinical pipeline for HIV/AIDS but only 8 for tuberculosis.
The cuts come at a time when many in the tuberculosis community feel that new treatments are within reach. In 2011, members of the TBTC published a paper demonstrating that the antibiotic rifapentine, sold as Priftin by France's Sanofi, could reduce the duration of treating latent tuberculosis from nine months of daily doses to three months of weekly doses (N. Engl. J. Med. 365, 2155-2166, 2011). This past May, at the American Thoracic Society's annual meeting in Philadelphia, Susan Dorman of the TBTC presented research suggesting that two months of treatment with rifapentine could yield sputum cultures clear of disease from people previously diagnosed with active tuberculosis.

The consortium, excited at the promise of rifapentine and the newly approved drug bedaquiline (sold by New Jersey's Janssen Pharmaceuticals as Sirturo; see Nat. Med. 18, 1157, 2012) had planned advanced trials for both drugs. However, in light of the sequester and likely future cuts to tuberculosis research, Schluger and other members of the consortium think these trials will be delayed by years, if not permanently.

In addition to the TBTC cuts, news reports have raised the possibility that tuberculosis research might suffer the further setback of a $\$ 45$ million funding cut in 2014 by the US Agency for International Development, which represents $20 \%$ of its budget for the disease.

Mario Raviglione, director of the World Health Organization's Stop TB Department in Geneva, thinks tuberculosis lacks the social and visceral pull that other maladies have when it comes to funding. "HIV is a disease that involves one of the most important aspects of human life-sex," he says. "Tuberculosis involves the sputum of poor people, and the poor are without voice in most societies."

Trevor Quirk 